\title{
Le Pragmatisme De Rorty : Entre Universalisme Et Relativisme
}

\author{
Désiré Médégnon (PhD) \\ Université d'Abomey-Calavi, Bénin
}

Doi: 10.19044/esj.2017.v13n29p202 URL:http://dx.doi.org/10.19044/esj.2017.v13n29p202

\begin{abstract}
Philosophy and science became what they are and developed because they have taken the universal both as the goal of research and the criteria of validity of every theory. For Richard Rorty, we have there a project with no foundation and totally unjustified. To Plato's universalism, Rorty opposes a pragmatism which defends philosophy as fitting as human being and considers that the only reasonable goal of research is to come to intersubjective agreements. In spite of the fact that it is difficult for science to get used to it, pragmatism has nevertheless done the great job of unveiling the illusion of an objective truth conceived as the speculum of reality.
\end{abstract}

Keywords: Pragmatism, universality, objectivity, intersubjectivity, conversation

\section{Résumé}

La philosophie et la science se sont constituées et développées en faisant de l'universel aussi bien le but de la recherche que le critère de validité de toute théorie. Pour Rorty, il s'agit là d'un projet infondé et totalement injustifié. A l'universalisme platonicien, Rorty oppose un pragmatisme qui défend une philosophie à hauteur d'homme et considère que le seul but raisonnable de la recherche est de parvenir à des accords intersubjectifs. Si la science peut difficilement s'en accommoder, le pragmatisme a, cependant, le mérité de démasquer l'illusion d'une vérité objective qui serait le speculum de la réalité.

Mots-clés : Pragmatisme, universalité, objectivité, intersubjectivité, conversation

\section{Introduction}

La quête de l'universel est apparue, au moins depuis Platon, comme une exigence incompressible de la philosophie et de la science (Gens, 1996 : 
251). Ramant à contre-courant de la tendance générale, Richard Rorty la considère cependant comme un projet infondé et inconsistant. Le pragmatisme dont il est l'un des porte-drapeaux les plus connus professe, aux lieu et place de cet «universalisme», une philosophie aux ambitions plus modestes, plutôt conformes à notre condition d'homme. De ce point de vue, le but de la recherche n'est plus de rechercher une vérité objective qui traduirait une correspondance avec la réalité, mais d'établir ou d'identifier, au détour de la conversation et du dialogue, laquelle des opinions ou croyances en présence est la meilleure. La question est alors de savoir si la science peut se satisfaire d'un tel projet ou si elle doit, malgré tout, faire de l'universalité, ne serait-ce qu'à titre asymptotique, le critère de validité des théories qu'elle travaille à établir.

\section{Pragmatisme ou relativisme ?}

On a souvent vu, dans la philosophie pragmatiste de Richard Rorty, une version moderne du relativisme. Tendance compréhensible et même parfaitement prévisible, dans la mesure où l'une des cibles privilégiées de la critique ou de la mise en perspective par laquelle le philosophe américain fonde son pragmatisme, c'est l'universalisme et, avec elle, l'idée qu'il existe une vérité objective, indépendante de l'homme et du procès par lequel celuici tente de l'établir, de le construire. Dans une communication présentée à Porto-Novo, voici bientôt une quinzaine d'années ${ }^{49}$, et publiée, d'abord dans Diogène ( $\mathrm{n}^{\circ} 203$, avril-juin 2003) puis dans La rationalité, une ou plurielle? (Rorty, 2007 : 11-24), le philosophe américain n'a pas hésité lui-même à prendre fait et cause pour Protagoras, à se ranger derrière lui et, de cette façon, à réaffirmer sa démarcation, sa distance vis-à-vis du projet et de la philosophie de Platon. Cette prise de position pourrait être interprétée, en effet, comme un aveu personnel de Rorty et considérée, à ce titre, comme un authentique élément de preuve pour valider l'équation "pragmatisme = relativisme ». Pourtant, assimiler le pragmatisme de Rorty au relativisme, ou même en faire une forme moderne et déguisée de relativisme, serait, en tout cas au regard de la profession de foi et des mises au point vigoureuses faites par l'auteur, sinon un mauvais procès, du moins le résultat d'une déduction hâtive. En effet, Rorty n'a jamais fait l'aveu de relativisme; bien au contraire, il le rejette, très clairement, "comme tout le monde », car de toute façon, l'idée que cette doctrine est censée incarner lui paraît tout simplement inadmissible et même, à la limite, insensée. Curieuse posture que celle de Rorty, dira-t-on. Comment comprendre, en effet, qu'il réfute le relativisme et

${ }^{49}$ C'était à l'occasion d'un colloque international qui s'est déroulé à Porto-Novo, au Bénin, du 19 au 21 septembre 2002, sur le thème : «La rencontre des rationalités ». Les actes de ce colloque ont été publiés, cinq ans plus tard, aux éditions du Codesria, sous le titre $L a$ rationalité, une ou plurielle? (Hountondji, 2007). 
que, dans le même temps, il critique si vigoureusement le projet qui en incarne la négation? Est-il possible et cohérent de rejeter le relativisme sans valider l'exigence de l'universel ?

Essentiellement portée par un souci de démarcation et de clarification, la réfutation du relativisme par Rorty a pris des formes différentes, quoique, dans tous les cas, l'objectif était de montrer qu'on ne saurait confondre pragmatisme et relativisme. Dans Conséquences $d u$ pragmatisme, Rorty part de la définition de la notion même du relativisme pour en montrer l'inconsistance et l'irrecevabilité indiscutables. Pour lui, et c'est un peu la ligne de défense qu'on découvre au cœur de son argumentation, le relativisme n'existe pas vraiment; et, conséquence directe de cette assertion, il n'existe pas un seul relativiste réel au monde. Pour faire court, le relativisme est un danger plutôt imaginaire, fictif. Et pour cause, par définition, le " "relativisme" est la conception selon laquelle toute croyance, pour une question donnée, voire pour quelque question que ce soit, est aussi bonne que n'importe quelle autre » (Rorty, 1993 : 309). Une représentation si ridicule qu'aucun esprit sérieux ne la validerait: «A l'exception de l'éventuel débutant de service, on ne trouvera personne qui accepte, sur une question importante, de tenir pour également bonnes, deux opinions incompatibles » (Rorty, 1993 : 309 ). L'expression de "relativisme' ne répond donc à aucune réalité ni concept, mais relève d'un onirisme, peutêtre, plus exactement d'une obsession, celle de l'universel, du moins tel qu'il est perçu par Platon et ses disciples plus ou moins proches - qu'ils s'appellent Descartes, Husserl ou Kant -, et de la nécessité de trouver un adversaire indéfendable dont la réfutation légitimerait la quête. L'accusation de relativisme serait donc une accusation infondée pour la simple raison que le délit référé n'a jamais pu et ne pourrait jamais exister, qu'il est plutôt une invention destinée à "marquer des points philosophiques" (Rorty, 1993 : 310), un « jeu » qui consiste à jouer avec des adversaires imaginaires plutôt qu'avec des interlocuteurs de travail réels :

«Le relativiste qui prétend que seules des considérations "non rationnelles" ou "non cognitives" permettent de départager des candidats à la fois sérieux et incompatibles à la croyance n'est jamais qu'un de ces petits copains imaginés par les platoniciens et les kantiens, qui partagent le même univers de fantasmes que le solipsiste, le sceptique et le nihiliste moral » (Rorty, 1993 : 310).

Dans Science et solidarité, la démarche de Rorty est plus pédagogique, et son analyse plus détaillée, plus nuancée, moins expéditive. Tout se passe comme s'il tentait de comprendre l'assimilation du pragmatisme au relativisme ; tout se passe comme s'il tentait de découvrir ce qui, mis à part la mauvaise foi, peut justifier ou, à défaut, expliquer la confusion que ses adversaires entretiennent au sujet du pragmatisme. Un des 
reproches qui sont faits au pragmatisme, constate Rorty, c'est de réduire l'objectivité à l'intersubjectivité. Et malheureusement, affirme-t-il, cela suffit pour accuser le pragmatiste de relativisme: «Il suffit d'assimiler l'objectivité à l'intersubjectivité pour être immédiatement accusé de relativisme. Telle est en effet l'épithète traditionnellement appliquée aux pragmatistes » (Rorty, 1990: 51). La question est alors de savoir si l'accusation est fondée, et si cette «épithète » correspond vraiment à l'attitude et à la posture du pragmatiste. Examinant donc la posture incriminée, à savoir l'assimilation de l'objectivité à l'intersubjectivité, Rorty fait remarquer qu'elle peut donner lieu à trois interprétations, à trois perceptions différentes dont une et une seule correspond à l'attitude et à la vision pragmatistes. La première, dont l'inconsistance saute aux yeux, renvoie à l'idée «stupide » selon laquelle toutes les croyances se valent. La deuxième revient à penser que « vrai est un terme équivoque qui a autant de significations que de contextes de justification ». Celle-ci est, comme la première, indéfendable et donc irrecevable. Reste la troisième, la toute dernière : elle repose sur la vision « ethnocentrique » qui enseigne qu'il n'y a rien à dire sur la vérité et la rationalité en dehors du tableau des «procédures familières $^{50}$ de justification » utilisées dans les différents domaines de recherche. Rorty reconnaît que le pragmatiste adopte bien cette conception ethnocentrique. Mais il s'empresse de préciser que « relativisme » n'est pas un terme approprié pour désigner cette sorte d'ethnocentrisme (Rorty, 1990 : 51).

Du texte que Rorty a présenté à Porto-Novo, nous nous référons à la traduction française parue dans La rationalité, une ou plurielle? Son importance est capitale dans la mesure où il est l'un des plus récents et qu'il donne à l'auteur l'occasion de repréciser sa théorie visiblement mal comprise et, surtout, de répondre, une fois encore, une dernière fois peut-être, à ceux qui, comme Habermas, l'accusent de relativisme. Nulle part, Rorty n'y établit une équivalence, encore moins une synonymie, entre le pragmatisme et le relativisme. La prise de position "derrière Protagoras", annoncée dans ce texte, et qu'on pourrait brandir pour confondre Rorty et fonder une telle équivalence, relève très précisément d'une démarche volontairement provocatrice, plus exactement pédagogique et, finalement, pragmatique, dont le but n'est pas de justifier ni de valoriser le relativisme, mais plutôt, et sur la base d'une lecture plus débridée et plus audacieuse de Protagoras, de faire découvrir (en ramant à contre-courant de la tendance générale), la cohérence et la consistance d'une posture et d'une maxime dont Platon a fait sa cible de choix et contre laquelle il s'est acharné d'un bout à l'autre de son œuvre. Il s'agit, dans le fond, de montrer les incohérences du rêve platonicien de

\footnotetext{
${ }^{50} \mathrm{C}$ 'est nous qui soulignons.
} 
l'universel, par une mise en perspective de la péjoration théorique dont a été victime Protagoras et sur la base de laquelle Platon a inventé la philosophie et en a fixé, pour ainsi dire, les conditions de possibilité tout autant que la nature. Que la philosophie procède ou doit nécessairement procéder de la négation de la posture de Protagoras, voilà précisément le mythe auquel s'attaque Rorty pour faire découvrir et tenter de corriger les excès du projet platonicien, selon lui, démesurément ambitieux. Rorty écrit, en effet :

"Vingt-quatre siècles plus $\operatorname{tard}^{51}$, on nous met toujours en garde contre les tentations du relativisme ${ }^{52}$, en nous exhortant pour le bien de la civilisation à venir, à nous ranger du côté de Platon. Convaincu cependant qu'il n'y a rien de tel, pour corriger les pratiques humaines, qu'un propos imaginatif qui suggère des pratiques humaines alternatives ${ }^{53}$, je me range pour ma part, du côté de Protagoras. Je vais donc proposer ici, comme je l'ai fait par le passé, une façon de concevoir le progrès moral et intellectuel qui s'accorde avec la maxime de Protagoras » (Rorty, 2007 : 11).

Rorty part de deux distinctions faites par Habermas et qui lui paraissent décisives. Nous n'évoquerons ici que l'une d'entre elles, à savoir celle qui oppose «la raison comme relation supposée de co-naturalité entre sujet et objet et la raison comme ensemble de pratiques sociales » (Rorty, $2007:$ 12).

L'idée d'une raison conçue comme relation de «co-naturalité entre sujet et objet » ou, ce qui revient au même, l'idée d'une raison centrée sur le sujet (subject-centered reason), reviendrait à considérer que les hommes possèdent la faculté d'aller directement à la connaissance, autrement dit, sans passer par la médiation de la conversation. Pour Rorty, évidemment, cette conception de la rationalité est une illusion, une absurdité à laquelle il faut tourner le dos, entendu que la raison réfère au dialogue et à la communication. La préférence du philosophe américain va donc, comme d'ailleurs celle de son collègue allemand, à la «raison communicationnelle ». A vrai dire, le terme de préférence est faible, car en fait c'est le seul choix qui s'impose. Et pour Rorty, ce choix est logiquement solidaire du renoncement à l'idée fantaisiste d'une vérité qui est censée traduire une adéquation entre le discours et la réalité. Réduite à son expression réelle, la vérité n'est et ne peut être que le résultat d'une recherche libre et imaginative qui met en scène ou qui implique des sujets engagés dans la "compétition sur le marché des idées", une «rude compétition entre des théories, des mouvements et des écoles alternatifs

\footnotetext{
${ }^{51}$ Vingt-quatre siècles après Platon.

${ }^{52}$ Ce n'est pas la première fois que Rorty fait cette remarque, visiblement suscitée par un agacement. En 1990, il écrivait déjà : « On nous met constamment en garde contre le danger du "relativisme" qui nous guette.» (Rorty, 1990b : 50).

${ }^{53}$ C'est nous qui soulignons.
} 
(Rorty, 1990 : 54). C'est cette conclusion que Rorty reproche à Habermas de n'avoir pas tirée. Quand ce dernier estime que, par sa prise de position, il "'s'aventure trop loin en direction du relativisme', Rorty s'en défend, bien sûr, et considère que le philosophe allemand fait une concession regrettable à l'universalisme. L'inconsistance démontrée de l'idée d'une « raison centrée sur le sujet » aurait dû conduire Habermas, logiquement, à abandonner la notion de validité universelle. S'il n'a pas porté à l'universalisme platonicien le coup fatal auquel préparait et invitait, logiquement, la substitution d'une conception communicationnelle à une conception de la raison centrée sur le sujet, c'est précisément du fait d'être resté, en réalité, prisonnier de cette dernière. Or, justement, pour Rorty, pas plus que le relativisme ne renvoie à rien de possible, l'universalisme ne saurait tenir lieu de doctrine sérieuse, les conditions de son effectivité étant, tout simplement, de l'ordre de l'improbable. Renvoyant ainsi dos à dos l'universalisme et le relativisme, Rorty propose une thèse qui, s'appuyant autant sur les vertus de la conversation que sur l'impossibilité de s'en passer, ramène les prétentions de la raison à des proportions raisonnables. Cette posture a un nom: le pragmatisme.

\section{Justification et concept : de la « Philosophie » à la « philosophie »}

Le pragmatisme est certainement une théorie révolutionnaire et même contestataire. Sans qu'on puisse l'assimiler au postmodernisme, le pragmatisme s'inscrit bien dans ce mouvement de déconstruction qui vise, d'une manière ou d'une autre, à s'interroger sur les certitudes et l'espoir incarnés et portés par les lumières, mouvement qui demande de relativiser, de revoir à la baisse, pour ainsi dire, les prétentions et les ambitions de la raison, et plus globalement de l'esprit humain. La critique rortienne de Platon et de son projet philosophique, la mise en perspective de l'exigence d'universalité dont il a fait la pierre angulaire de sa philosophie et même de la philosophie, de façon générale, participe d'un besoin de redimensionnement de l'espace de validité du discours et de la pensée, que ceux-ci portent sur les valeurs morales ou qu'ils réfèrent à la connaissance. Et si, dans la perspective de ce redimensionnement, de cette révision du « champ du possible », Platon reçoit un traitement spécial, ce n'est pas seulement du fait d'être le fondateur de l'universalisme; c'est aussi et surtout d'avoir installé la fausse alternative « universalisme-relativisme » et, ainsi, de laisser croire que le rejet de l'un des termes entraîne nécessairement l'adoption et la consécration de l'autre, et vice-versa. La longue réfutation du relativisme (aussi bien dans Science et solidarité que dans Conséquences $d u$ pragmatisme) alors même que sa véritable cible est ailleurs, la tentative renouvelée, chaque fois que l'occasion lui en est donnée, de distinguer le pragmatisme du relativisme, d'avoir à dire et à redire que le pragmatisme 
n'est pas relativisme, qu'il ne peut être relativisme, est pour Rorty, une tâche passablement agaçante, mais à laquelle il ne peut se dérober. Cet effort incessant de clarification se justifie et s'impose en raison, précisément, de l'illusion générée par l'apparente transparence de l'alternative « universalisme ou relativisme » qui rappelle, soit dit en passant, par son caractère caricatural, celle par laquelle Engels réduisait à deux et pas plus, les conceptions fondamentales qu'on pouvait avoir du monde, à savoir le matérialisme et l'idéalisme. La tentative de Richard Rorty semble vouloir corriger une erreur et dire une chose simple : la négation de l'universalisme, ou plus exactement la mise en perspective du projet d'une vérité objective et universelle, n'est pas nécessairement relativisme. Il faut sauter le verrou de cette hantise. Il faut dénoncer le caractère utopique de ce projet platonicien et libérer la philosophie de cette exigence mutilante et paralysante. C'est, selon Rorty, la seule et unique façon de la rendre à son dynamisme et à sa nature essentielle. C'est la seule voie pour la sauver du gaspillage de forces et d'énergies que l'on gagnerait à consacrer à autre chose qu'à la recherche de vérités prétendument idéales, objectives et universelles : «Pour les pragmatistes, si l'on veut donner toutes les chances à la philosophie, il faut s'interdire de pratiquer la Philosophie ${ }^{54}$.» (Rorty, 1993: 16). Il faut abandonner le projet de cette «Philosophie» avec grand « $\mathrm{P} »$, et aux ambitions tout aussi hors de portée qu'inutiles, pour une philosophie avec petit « p », aux ambitions plutôt modestes et honnêtes, à hauteur d'homme, une philosophie surtout utile et féconde. Il faut passer à la seule philosophie qui vaille la peine, la «philosophie non-spéculaire» (Rorty, 1990a : 392). Tel est, en substance, le projet qu'incarne le pragmatisme. Gilbert Hottois résume parfaitement donc le projet et le propos du philosophe américain lorsqu'il écrit : «R. Rorty nous parle, avant tout, de la philosophie : de ce qu'elle est, a été, devrait être et ne pas être. » (Hottois, 1994 : 7).

Et pour cause. Il est illusoire de penser, comme nous le suggère le platonisme, qu'il doit exister, au-delà de l'humanité, des normes éternelles fixées par une autorité infaillible et omnisciente, et donc au-dessus de tout soupçon et de toute corruption possibles, des normes valables en tout temps et en tout lieu, des normes en soi, qui transcenderaient ainsi la fragilité et la finitude de la condition humaine, mais que les hommes seraient, malgré tout, dans l'obligation morale de respecter. Une telle idée relève de l'imaginaire et, pour cette raison, ne saurait suffire à fonder la quête et l'exigence d'une vérité objective, reflet présumé de la réalité dans ce que cette dernière est censée avoir d'intrinsèque et donc d'indépendant du sujet qui l'aborde et en rend compte. Perd ainsi toute crédibilité et toute consistance, la «théorie

${ }^{54}$ Il faut abandonner le projet de cette «Philosophie » avec grand $\mathrm{P}$ et aux ambitions tout aussi hors de portée qu'inutiles pour une philosophie avec petit $\mathrm{p}$, aux ambitions plutôt modestes et honnêtes, à hauteur d'homme, une philosophie surtout utile et féconde. 
contemplative de la connaissance », comme la nomme plaisamment Dewey, c'est-à-dire l'idée d'une vérité qui serait la représentation et la correspondance de la réalité. Le but de la recherche ne peut être de converger vers un point unique, vers "une vérité qui, située "en dehors de nous", attendrait que les êtres humains la rejoignent » (Rorty, 1990b : 53).

Avec le pragmatisme de Rorty, c'est la naïveté enfin mise au jour et dépassée d'une philosophie spéculaire qui prétend, tel un miroir (speculum), renvoyer l'image de la réalité telle qu'elle est, sans altération. C'est le piège détecté et écarté de cet "excès d'assurance qui consiste particulièrement à penser qu'il peut exister, au sens de réalisme, une fidélité à la réalité » (Rorty, 1990a : 418). Obnubilés par l'idéal platonicien du vrai, nombre de philosophes sont tombés dans ce piège. Le cas le plus «désolant», pour Rorty, est probablement celui de Jürgen Habermas. Rorty est séduit par l'intuition géniale qu'a eue l'auteur du Discours philosophique de la modernité d'opposer à la conception vaporeuse d'une «raison centrée sur l'objet», celle de «la raison communicationnelle», la seule qui tienne la route et qui rende vraiment compte du vrai visage de la raison et de la rationalité. Visiblement, cette intuition a tourné court faute d'avoir perçu ses propres prolongements théoriques. Si la vocation de la rationalité est de se déployer dans un cadre dialogique, d'être une « rationalité de l'entente », une rationalité «procédurale » qui s'incarne dans la pratique de l'argumentation (Habermas, 2001 : 17), cela suppose que les énoncés ou assertions ne sont fondés qu'en ce qu'ils peuvent être critiqués et justifiés. Dans le fond, Rorty ne dit pas autre chose. Mais, si les deux philosophes s'accordent sur la vocation de la raison et sur son terrain d'exercice, si l'intersubjectivité est à la fois le lieu et le critère de validation de tout énoncé, si, enfin, ce qu'il est convenu d'appeler la vérité ne se donne que dans le cadre de la conversation, c'est au sujet des implications et de la signification de l' «entente » ou de l' «accord » des sujets que Habermas et Rorty se démarquent l'un de l'autre. Habermas insiste en effet pour dire que le destin de la discussion que commande la «rationalité communicationnelle » n'est pas de s'accommoder d'une juxtaposition muette des théories en compétition, mais de parvenir à une entente. Mais cette entente suppose, pour le philosophe allemand, l'existence de quelque chose qui dépasse le cadre de chaque sujet pris isolément, quelque chose qui se trouve hors de chaque sujet, et auquel tout le monde se branche, comme à une source commune et unique. On pourrait dire, à cet effet, que pour Habermas, l'intersubjectivité est le lieu et la figure dans lesquels l'objectivité se manifeste et se révèle :

«Cette rationalité de la communication trouve son expression dans la capacité du discours orienté vers l'entente à favoriser l'accord, ce discours garantissant aux locuteurs qui y participent à la fois l'existence d'un monde 
vécu, intersubjectivement partagé et l'horizon à l'intérieur duquel ils peuvent se référer à un seul et même monde objectif » (Habermas, 2001 : 49).

C'est précisément la référence à ce «monde objectif » que Rorty trouve absolument fantaisiste et inacceptable, pour la simple raison qu'elle relève d'une extrapolation, pour le moins, abusive. Pour Rorty, en effet, l'accord intersubjectif ne présuppose en rien l'existence d'une quelconque référence extérieure au sujet ou à la discussion. Ce qui fonde cet accord, c'est l'opportunité ; ou si l'on préfère, ce qui la rend possible, c'est la tolérance, car justement, parvenir à un accord ne supprime pas nécessairement les désaccords.

Il serait cependant erroné de penser que Rorty refuse toute idée de vérité ou d'objectivité, ou que ces deux notions sont incompatibles avec le pragmatisme. Ce que rejette Rorty, ce ne sont pas les notions en ellesmêmes, mais plutôt le contenu et la signification qu'on leur donne. Si l'on est en droit de parler de vérité, et Rorty n'en doute point - bien au contraire -, l'erreur vient de ce qu'on l'assimile à l'image, au double idéel de la réalité. Rorty est profondément d'accord avec les platoniciens que le critère et le fondement de ladite vérité n'est autre que l'objectivité ; mais c'est sur la définition et le référentiel de cette objectivité qu'il s'oppose à eux. La vérité n'est pas «contact avec la réalité », mais «ce que nous avons de mieux à croire ». Ce «mieux », cette meilleure opinion est déterminée, évidemment, dans et par le jeu de la conversation.

Finie donc l'illusion de recherches dont on attend qu'elles nous donnent, sous la dictée de la réalité, une copie qui en soit l'exacte réplique. De ce type de recherches, la science était censée être le prototype, le modèle accompli. On a tôt fait, en effet, d'élever cette dernière au statut d'une «discipline qui offre une vérité "objective", "dure" : la vérité qui correspond à la réalité, la seule qui soit digne du nom » (Rorty, 1990 b : 46). Une telle conception relève, aux yeux de Rorty, d'une méprise si ce n'est le résultat d'une dilatation, d'un grossissement du pouvoir de la science et, bien entendu, de ceux qui l'animent. C'est à croire qu'il existe, au-delà de l'humanité, des vérités que l'homme de science peut déceler et révéler, grâce à un pouvoir spécial dont il serait investi et qui le disposerait à franchir le fossé entre l' « humain » et le « non-humain »: «Les hommes de science ont remplacé les prêtres » (Rorty, 1990b : 46).

Dépassée aussi, dans la même logique, la distinction tranchée et mécanique, l'opposition systématique et irréductible que l'on a cru devoir installer entre l'objectif et le subjectif. Sans renoncer au principe de l'objectivité, Rorty demande cependant qu'elle soit ramenée à ce qu'elle a toujours été, à la seule chose qu'elle peut être : un « accord intersubjectif », ni plus ni moins. La seule manière raisonnable et honnête de concevoir l'objectivité, si tant est que ce mot est censé avoir un sens, c'est de 
l'assimiler à l'intersubjectivité. Cette intersubjectivité a un mode d'emploi que Rorty présente, de manière limpide : «Le désir d'objectivité n'est que le désir d'accéder à des croyances qui feront éventuellement l'objet d'un accord sans contrainte lors d'un débat libre et ouvert à ceux dont les croyances sont différentes » (Rorty, 1990b : 58-59). Vole ainsi en éclats la thèse de l'inconsistance théorique de l'opinion défendue par Platon (Cf. Théétète, 1, 146 c-d) mais également par Gaston Bachelard ${ }^{55}$. Volent en éclats, par la même occasion, les dichotomies «opinion-vérité » et «croyances-savoirs ». Dans le cadre de la conversation, sur le terrain privilégié et incontournable du dialogue, les hommes peuvent exprimer leurs croyances ou leurs opinions respectives, les confronter, les mesurer les unes aux autres et, à la fin (une fin nécessairement provisoire), déterminer laquelle est la plus intéressante, la plus utile, la plus économique, la plus féconde. Dans cette quête, évidemment, il n'y a pas d'a priori ni de préjugé à faire valoir, entendu que seuls la conversation et le débat sont susceptibles d'établir le degré de pertinence et de fécondité de l'opinion, et que la manière la plus sûre de savoir ce qu'on doit croire, est de se montrer ouvert au plus grand nombre possible de propositions et d'arguments.

Rorty peut alors, en tournant dos à une tradition philosophique et scientifique qui remonte à Platon, proposer une autre, moins ambitieuse, moins exigeante sans doute aussi, mais plus cohérente à ses yeux et à ceux des pragmatistes; il peut redéfinir le but de la recherche: c'est «la possibilité de parvenir à une combinaison opportune, faite d'accord sans contrainte et de désaccord tolérant où ce qui est opportun est déterminé à coups d'essais et d'erreurs » (Rorty, 1990b : 58). Valable pour toutes les sphères de la culture, ce but signifie, lorsqu'on l'arrime à l'univers particulier de la science, au moins deux choses. Tout d'abord, on ne saurait exiger d'une théorie scientifique qu'elle mette d'accord tout le monde, ce qui veut dire que l'existence de désaccord ne l'invalide pas d'office. Deuxième chose : contrairement à ce qu'on pense et dit en général, c'est plutôt l'opportunité qui définirait et déterminerait aussi la théorie scientifique. Le mode de détermination même de cette opportunité (à "coups d'essais et d'erreurs") indique clairement que le repère qui valide la théorie ne saurait être un quelconque principe extra-humain, soustrait à la fragilité et la

55 L'opinion, chez Bachelard, n'est pas seulement frappée de nullité et d'inconsistance théoriques. Elle est même considérée comme un obstacle épistémologique : « La science, dans son besoin d'achèvement comme dans son principe, s'oppose absolument à l'opinion... L'opinion a, en droit, toujours tort. L'opinion pense mal ; elle ne pense pas : elle traduit des besoins en connaissances. En désignant les objets par leur utilité, elle s'interdit de les connaître. On ne peut rien fonder sur l'opinion: il faut d'abord la détruire. Elle est le premier obstacle à surmonter » (Bachelard, $1977: 14)$. 
fugacité de nos conditions et de notre monde, mais plutôt l'efficacité, l'utilité.

\section{Défis}

Au regard des repères qui, jusqu'ici, ont guidé les recherches aussi bien en philosophie qu'en science et dans bien d'autres domaines de la culture humaine, le pragmatisme, tel qu'il est présenté et défendu par Richard Rorty, représente, indiscutablement, un véritable défi. En effet, renoncer au principe de l'objectivité ou, ce qui revient à peu près au même, le réduire à l'intersubjectivité et, de cette façon, postuler et affirmer que la quête de vérités universelles n'est ni fondée, ni utile, à plus forte raison, c'est tourner le dos aux principes qui ont toujours guidé les inlassables efforts d'intelligibilité du réel, sous tous ses aspects, et qui expliquent, pour une bonne part, l'efficacité pratique et la fécondité des savoirs établis, même si ces derniers sont provisoires et appelés à être affinés. Réduire l'aspiration et la vocation du jugement humain à n'être qu'un avis parmi tant d'autres, une croyance concurrentielle d'autres, une opinion au milieu d'autres opinions, et dont la validation ne signifie rien d'autre qu'il est ce qu'il y a de mieux à penser et à faire, n'est-ce pas, au fond, manquer d'ambition et injustement affirmer, ad vitam aeternam, l'incapacité de l'homme à connaître ce qui est et savoir ce qui doit être?

C'est essentiellement sur le terrain de la science et de la technologie que le pragmatisme de Rorty pose problème. Si le philosophe américain avait raison, si l'on doit admettre que la théorie scientifique ne peut prétendre à la vocation de l'universel, comment comprendre que des savants ou chercheurs travaillant sur le même sujet parviennent, sans se concerter, sans se connaître, à des résultats identiques ? Comment expliquer cette convergence, si l'on persiste à croire qu'il est vain de postuler, comme le fait Habermas, «l'existence d'un monde vécu intersubjectivement partagé », si l'on ne la réfère pas à «un seul et même monde objectif » indépendant du sujet, un monde qui attend d'être découvert, et dont la théorie a vocation de rendre compte ? (Habermas, 2001 : 49).

Il semble, tout de même, que les succès obtenus dans le projet de mise en formule du réel n'ont été possibles, dans une large mesure, que parce que la science s'est investie du rôle de traquer ledit réel et d'en rendre compte avec fidélité. De ce point de vue, elle ne pouvait, en aucun cas, s'accommoder de relativisme. Et on comprend qu'après Platon et les autres, Husserl le rejette systématiquement, et surtout, que ce rejet lui permet de fonder, en raison, l'unité et l'objectivité du savoir scientifique (Husserl, 1969 et Husserl, 1976). On comprend que Rorty lui-même s'inscrive ouvertement dans la logique de ce rejet. La réfutation, la longue et laborieuse réfutation du relativisme à laquelle il s'est consacré est, à y regarder de près, la preuve 
que le débat contradictoire ou la «compétition des idées » dont la conversation est aussi bien le lieu que le moyen, ne peut fonctionner et prospérer que si les interlocuteurs concernés peuvent, jusqu'à un certain point, référer à la même chose, à un même objet, à un même monde.

Et pourtant, l'appel de Rorty, la critique qu'il développe dans le cadre du pragmatisme, doit être prise très au sérieux. Il serait excessif, en effet, de considérer que le pragmatisme marque une régression et un recul. Richard Rorty a tout à fait raison lorsqu'il trouve prétentieux de présenter la vérité scientifique comme le «double imagé », le speculum de la réalité. Cette conception reviendrait, ni plus, ni moins, à étendre au-delà de ses limites réelles, quoique provisoires, l'aire de validité des théories scientifiques. Connues pour être provisoires, celles-ci ne peuvent être, dans tous les cas, que des connaissances approchées d'une réalité posée comme repère. Résultat: le savoir scientifique le plus établi n'est, à tout prendre, que « la meilleure théorie disponible », en attendant d'être dépassé par un autre auquel le même sort est réservé. Les théories ou les croyances en cours, la nature même des objets accessibles à l'analyse, l'équipement pour les étudier, qu'il s'agisse de l'équipement technique ou de l'arsenal mathématique, la façon d'observer ces objets et d'en parler, voilà autant de paramètres qui affectent la connaissance scientifique et dont la prise en compte interdit logiquement de valider l'équation: "vérité = adéquation avec la réalité ».

L'histoire de la physique nous fournit d'ailleurs des exemples édifiants. Nous en évoquerons deux. Le premier concerne la nature « réelle » des particules microphysiques. La question de savoir si ces dernières sont des corpuscules ou plutôt des ondes, question difficile à trancher, entendu que la réponse dépend du matériel choisi ${ }^{56}$ pour l'observation, a amené bon nombre de physiciens à abandonner, à leur corps défendant, les notions de « réalité indépendante de l'observateur » et d' «objectivité ». L'impossibilité, par ailleurs, de pouvoir déterminer, de façon simultanée, la vitesse et la position des particules quantiques, impossibilité établie par les "relations d'indétermination', a amené à renoncer au principe du déterminisme scientifique $^{57}$. Il n'en fallait pas plus pour susciter cet avis de John Von Neumann :

\footnotetext{
${ }^{56} \mathrm{C}$ 'est nous qui soulignons

${ }^{57}$ En dépit de la réserve émise par quelques physiciens, cette nouvelle théorie a fini par s'imposer. Dans Heurs et malheurs de la physique quantique : des vérités incroyables JeanPierre Pharabod et Gérard Klein reviennent sur les difficultés et les polémiques qui ont marqué l'histoire de la physique cette physique, plus précisément, sur le désaccord entre l'Ecole de Copenhague et les physiciens regroupés autour d'Einstein (Pharabod et Klein, 2017)
} 
«Les sciences n'essaient pas d'expliquer; c'est tout juste si elles tentent d'interpréter; elles font essentiellement des modèles. Par modèle, on entend une construction mathématique qui, à l'aide de certaines interprétations verbales, décrit les phénomènes observés. La justification d'une telle construction mathématique réside uniquement et précisément dans le fait qu'elle est censée fonctionner » (Neuman, 1989 : 343).

Le second exemple, un peu dans le même registre, est relatif à la nature de la lumière. Deux théories, toutes parfaitement opérationnelles, ont pu cohabiter: la théorie corpusculaire défendue notamment par Newton et Einstein, et la théorie ondulatoire que nous devons à Maxwell. L'impossibilité de dire laquelle des deux théories traduit l' "adéquation avec la réalité » nous porte à les considérer comme deux lectures d'un seul et même fait, et surtout à considérer que le recours à l'une d'entre elles, dans une occurrence donnée, ne signifie par autre chose qu'elle est, en cette occurrence précise, la «meilleure » des options.

Le fait qu'un même fait, qu'une même situation, donne lieu à plusieurs théories, pourrait être considéré non comme un problème, mais plutôt comme une chance. Il doit être accepté comme l'expression, non d'un relativisme, mais plutôt d'une ouverture qui, si elle est bien assumée, maximiserait les possibilités de décryptage et de connaissance d'un réel plutôt complexe et qui ne s'épuise pas dans une vérité, fût-elle scientifiquement établie.

\section{Conclusion}

Aux yeux de Richard Rorty, l'idéal platonicien de vérité et d'universalité a l'inconvénient de détourner la philosophie du possible et de l'utile. En renonçant à cet idéal, le philosophe américain ne croit cependant pas tomber dans le piège du relativisme. Le pragmatisme dont il se réclame considère que la seule tâche qu'on peut raisonnablement assigner à la recherche, quel que soit le domaine référé, est de conduire les hommes qui s'y consacrent, par le jeu de la conversation et de la compétition des idées, à des accords intersubjectifs, sans autre prétention. On peut comprendre que les adversaires du pragmatisme y voient une démarche peu ambitieuse et même une régression regrettable. Mais, que la science et la philosophie définissent et dimensionnent leurs ambitions sur la base du contexte réel de la recherche, ne serait-ce pas, après tout, un gage de performance et d'efficacité ? 


\section{References:}

1. Bachelard, G. (1977). La formation de l'esprit scientifique. Paris : Vrin.

2. Gens, J.-C. (1996). Richard Rorty et le spectre du relativisme. Hermès, 20,

3. pp. 251-258.

4. Gleick, J. (1989). La théorie du chaos, trad. J. Mougin, Paris : Albin Michel.

5. Habermas, J. (1988). Le discours philosophique de la modernité. Douze conférences, trad. Christian Bouchindomme \& Rainer Rochlitz. Paris : Gallimard.

6. Habermas, J. (2001). Vérité et justification, trad. Rainer Rochlitz. Paris : Gallimard.

7. Hottois, G. (1994). Présentation. Dans G. Hottois \& M. Weyembergh (Dir.), Richard Rorty: ambiguités et limites du postmodernisme (pp. 7-12). Paris : Vrin.

8. Husserl, E. (1976). L'origine de la géométrie, trad. J. Derrida, dans La crise des sciences européennes (pp. 411-427). Paris : Gallimard.

9. Husserl, E. (1969). Recherches logiques, Trad. H. Elie, tome $1^{\mathrm{er}}, 2^{\mathrm{ème}}$ éd. Paris : PUF.

10. Pharabod, J.-P. \& Klein, G. (2017). Heurs et malheurs de la physique quantique : des vérités incroyables. Paris : Odile Jacob.

11. Platon (1995). Théétète, trad. M. Narcy, Paris : Flammarion.

12. Rorty, R. (1990a). L’homme spéculaire, trad. Th. Marchaise, Paris : Seuil.

13. Rorty, R. (1990b). Science et solidarité. La science sans le pouvoir, trad. J.-P. Commeti, Paris : Editions de l'Eclat.

14. Rorty, R. (1993). Conséquences du pragmatisme, trad. J.-P. Commeti, Paris : Seuil.

15. Rorty, R. (2007). Hauteur universaliste, profondeur romantique, ruse pragmatique. Dans P. J. Hountondji (Dir.), La rationalité, une ou plurielle? (pp. 11-24). Dakar: CODESRIA. 